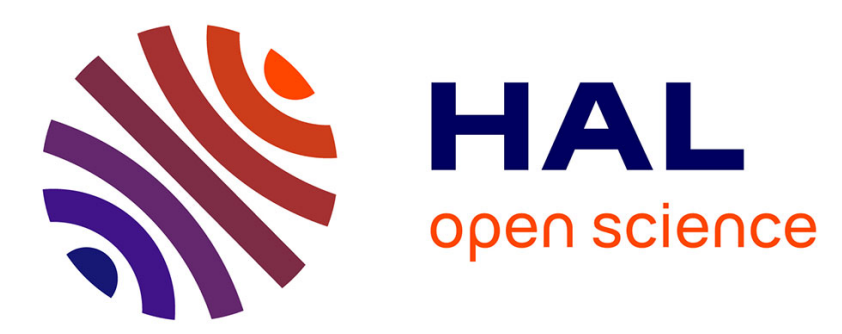

\title{
Dmipy, a Diffusion Microstructure Imaging toolbox in Python to improve research reproducibility
}

\author{
Abib Alimi, Rutger H.J. Fick, Demian Wassermann, Rachid Deriche
}

\section{To cite this version:}

Abib Alimi, Rutger H.J. Fick, Demian Wassermann, Rachid Deriche. Dmipy, a Diffusion Microstructure Imaging toolbox in Python to improve research reproducibility. MICCAI 2018 - Workshop on Computational Diffusion MRI, Sep 2018, Granada, Spain. hal-01873353

\section{HAL Id: hal-01873353 \\ https://hal.inria.fr/hal-01873353}

Submitted on 13 Sep 2018

HAL is a multi-disciplinary open access archive for the deposit and dissemination of scientific research documents, whether they are published or not. The documents may come from teaching and research institutions in France or abroad, or from public or private research centers.
L'archive ouverte pluridisciplinaire HAL, est destinée au dépôt et à la diffusion de documents scientifiques de niveau recherche, publiés ou non, émanant des établissements d'enseignement et de recherche français ou étrangers, des laboratoires publics ou privés. 


\title{
Dmipy, a Diffusion Microstructure Imaging toolbox in Python to improve research reproducibility
}

\author{
Abib Alimi ${ }^{\star 1}$, Rutger Fick*2, Demian Wassermann ${ }^{3}$, and Rachid Deriche ${ }^{1}$ \\ 1 Athena, Inria, Université Côte d'Azur, Sophia Antipolis, France \\ 2 TheraPanacea, Paris, France \\ 3 Parietal, Inria, CEA, Université Paris-Saclay, Paris, France
}

\begin{abstract}
Non-invasive estimation of brain white matter microstructure features using diffusion MRI - otherwise known as Microstructure Imaging - has become an increasingly diverse and complicated field over the last decade. Multi-compartment-based models have been a popular approach to estimate these features. In this work, we present Diffusion Microstructure Imaging in Python (Dmipy), a diffusion MRI toolbox which allows accessing any multi-compartment-based model and robustly estimates these important features from single-shell, multi-shell, and multi-diffusion time, and multi-TE data. Dmipy follows a building block-based philosophy to microstructure imaging, meaning a multicompartment model can be constructed and fitted to dMRI data using any combination of underlying tissue models, axon dispersion-or diameter distributions, and optimization algorithms using less than 10 lines of code, thus helps improve research reproducibility. In describing the toolbox, we show how Dmipy enables to easily design microstructure models and offers to the users the freedom to choose among different optimization strategies. We finally present three advanced examples of highly complex modeling approaches which are made easy using Dmipy.
\end{abstract}

Keywords: diffusion MRI - microstructure imaging · dMRI - multicompartment models $\cdot$ python $\cdot$ free open source software.

\section{Introduction}

Diffusion MRI (dMRI) is sensitive to the micrometer-scale displacement of water molecules and has, therefore, become an invaluable clinical diagnostic tool, particularly in neuroimaging. In principle, dMRI can provide information about the tissue microstructure on a much smaller scale than that of the actual image resolution: micrometers versus millimeters.

The practice of estimating microstructural tissue features using dMRI is referred to as Microstructure Imaging. In Microstructure Imaging, the observed diffusion signal is related to tissue structure using biophysical models (see a partial taxonomy in [1).A combination of biophysical models constitutes a microstructure model. In particular, multi-compartment (MC)-based models have been a popular approach to estimate these features.

\footnotetext{
* Abib Alimi and Rutger Fick share first authorship for this work.
} 
Different toolboxes have been proposed so far. The Microstructure Imaging Sequence Simulation Toolbox (MISST) [2]3] applies a generalized gradient waveform to microstructure models and eventually estimates their parameters. However, the main goal of MISST is to accurately simulate the diffusion signal of microstructure models generally hard-coded in the toolbox. The recently introduced CUDA diffusion modeling toolbox (cuDIMOT) 4 proposes to use Graphical Processing Units (GPUs) in order to accelerate the computationally expensive biophysical modeling and microstructure mapping to diffusion measurements as well as tractography and connectivity estimation. In [5] the authors go further in term of reducing computation time in their Microstructure Diffusion Toolbox (MDT) in which they use parallelized GPUs and multi-core CPU in diffusion modeling in large group studies.

To provide access to any MC-based model and improve reproducibility in MC-based research, we propose Diffusion Microstructure Imaging in Python (Dmipy). Dmipy is a diffusion MRI toolbox allows accessing any MC-based model. It uses multi-core CPUs and modularity is a key feature that simplifies both its implementation e.g future extension and its usage. Furthermore, unlike the above-mentioned toolboxes, not only does Dimpy propose to use 'standard' MC-modeling strategy but also offers the spherical mean (SM) of any microstructure model, as well as a generalization of the Constrained Spherical Deconvolution (CSD) 6 technique and adds new tissue models to CSD and SM. Dmipy robustly estimates these models' important features from single-shell, multi-shell, and multi-diffusion time diffusion data, using less than 10 lines of code and therefore helps improve research reproducibility . It is an open source package currently available for download on https://github.com/AthenaEPI/dmipy/.

This paper extends our previous work [78] and gives a further explanation on the design of microstructure models and the optimization algorithms available in Dmipy to estimates microstruction features as illustrated in Fig. 1.

\section{Dmipy Signal Representation and Multi-Compartment Modeling}

\subsection{Diffusion contrast}

In this work, we focus on probing the tissue microstructure using the standard Pulsed Gradient Spin-Echo sequence (PGSE) 9] to obtain diffusion-weighted images (DWIs). The measurement of the diffusion signal is directly related to the concept of attenuation. In the presence of diffusion, the signal intensity $S(b)$ is lower than the non-diffusion-weighted signal $S(0)$. The signal attenuation is then expressed as $E(b)=S(b) / S(0)$. Without restricting boundaries in the diffusion process, the signal attenuation is then given as a Gaussian as $E(b)=\exp (-b D)$ with $b$ the b-value and $D$ the water diffusivity 9 .

It is possible, but impractical, to densely sample all of $E(\mathbf{q}, \tau)$ and nonparametrically recover the displacement probability $P(\mathbf{r}, \tau)$ [11], from which some information about the tissue structure can be inferred. A more efficient approach has been to study the geometry of different tissue types to come up 


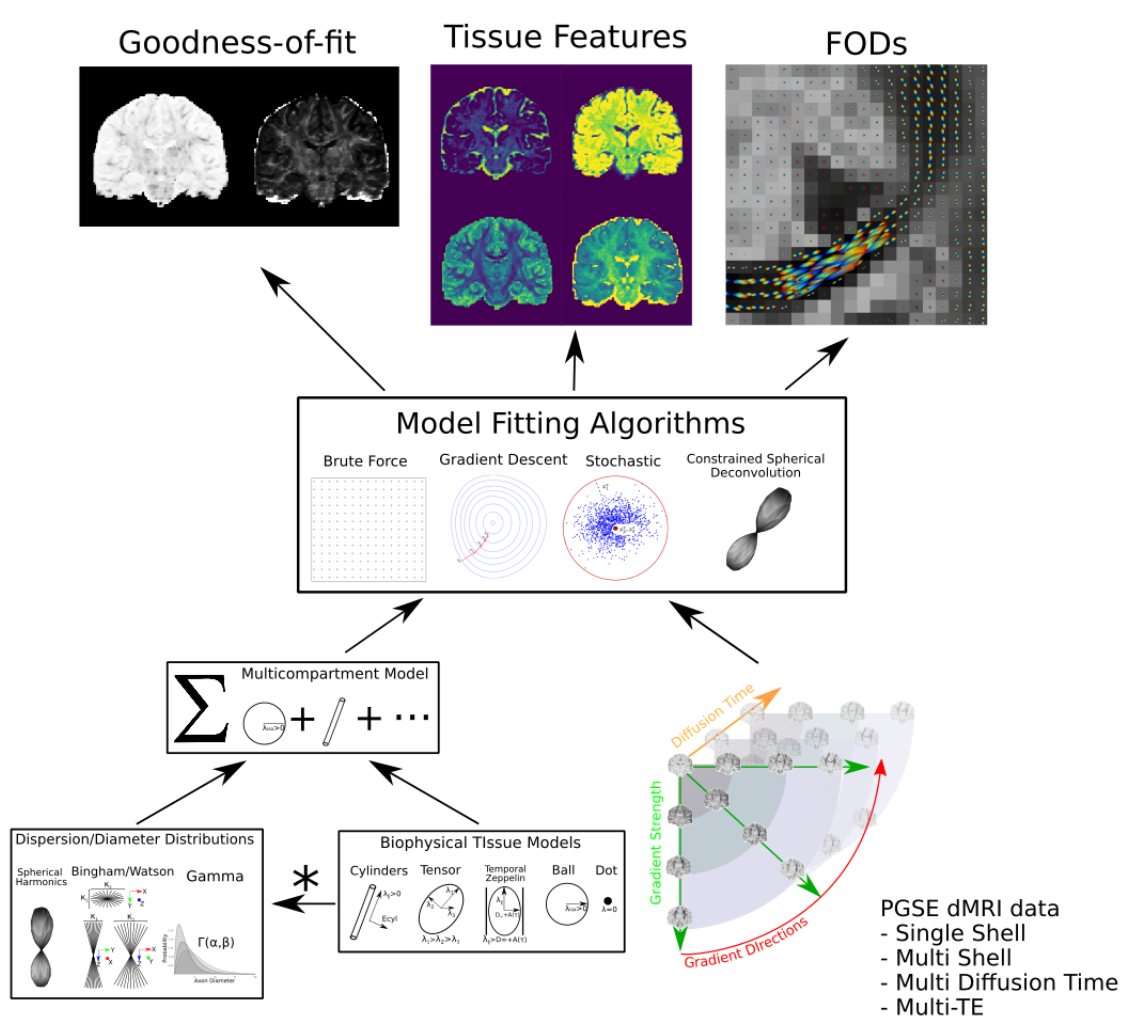

Fig. 1: Dmipy workflow: Modular microstructure model setup and parameter estimation. Different biophysical tissue models (see Fig. 22) are dispersed and/or distributed and combined together in a multi-compartment model, which is then fit to diffusion data using a chosen optimization algorithm to estimate tissue feature parameters, reconstruct FODs and quantify the quality of the fitting.

with mathematical representations which approximate the diffusion in those tissues. For example, diffusion inside axons with some radius is typically modeled as diffusion inside cylinders with the same radius, which has an analytical expression (e.g. [12]). Combinations of these representations can then be fitted to more sparsely measured DWIs, after which the recovered representation parameters (such as the diameter of the cylinder) are used to interpret the structure of the tissue. Such an approach is called multi-compartment modeling or Microstructure Imaging and is the focus of the Dmipy software project.

\subsection{Biophysical modeling}

Biophysical models are mathematical representations of different tissue types, based on assumptions found in cited references. Here we show an extensive overview of these models as illustrated in Fig. 2 . 


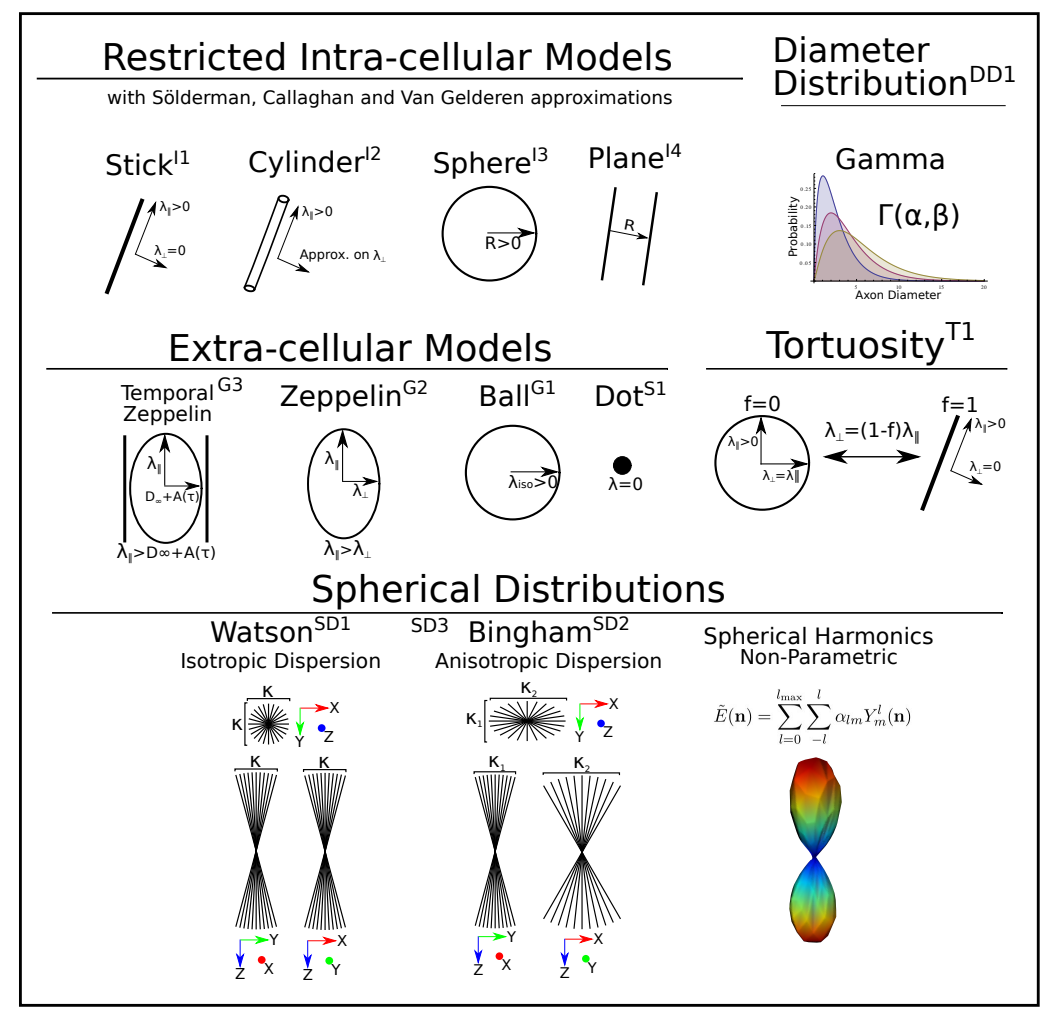

Fig. 2: A schematic of most biophysical models used in PGSE-based Microstructure Imaging. Using different combinations of these "components", any microstructure model can be assembled using Dmipy.

Models of Intra-cellular Diffusion (I1 through I4). Diffusion in the intracellular space is represented using axially symmetric and restricted geometric (cylinders, spheres, planes) models, with the orientation parallel to the axis. The threedimensional diffusion signal in these models is given as the separable product of parallel restricted perpendicular diffusions [13. For each geometry, Dmipy offers different approximations i-e Solderman, Gallaghan and Gaussian Phase approximations. More details can be found in references [14|15]17/12 18 19].

Axonal Diameter Distribution (DD1). Histology studies [20] showed that the diameters of the axons are not constant throughout the brain, but they follow a distribution. In Microstructure Imaging, the true axon diameter distribution is usually modeled by a Gamma distribution 21, which is implemented in Dmipy for the aforementioned restricted geometries.

Models of Extra-cellular Diffusion (G1 through G3). Hindered extra-cellular diffusion is often modeled as an anisotropic, axially symmetric gaussian called 
"Zeppelin" (G2) in [1. However, recent works argue that hindered diffusion is actually slower-than-exponential over diffusion time $\tau$ due to how the external axon boundaries still hinder diffusing particles 23. Later on, 24] proposed a modification of the Zeppelin taking into account the time-dependence of the diffusion, which Dmipy dubbed the temporal zeppelin (G3).

Parameter links Note that our toolbox enables to relate the volume fractions of the different compartments by modeling a first-order tortuosity (T1) approach to modulate their diffusivities as in [26].

Models of Axonal Orientation Distributions (SD1 through SD3). Histology studies show that the orientation of axons within one white matter bundle are dispersed around the central bundle direction [25]. Therefore, the diffusion signal can be modeled as the spherical convolution of a spherical distribution and a kernel which describes the signal of a single axon micro-environment. Dmipy models this spherical distribution using, as most generally, the spherical harmonics (SD3) functional basis 28 29] to give the so-called fiber Orientation Distribution Function (fODF) or Fiber Distribution Function (FOD). The toolbox also provides Bingham (SD2) and Watson (DS1) distributions [31|32|22], more straightforward to represent anisotropic and isotropic dispersions, respectively.

\section{$2.3 \quad$ Multi-compartment modeling}

Using Dmipy, any selection of biophysical models can be combined into a multicompartment model, of which the framework provides three different types:

- multi-compartment (MC) or 'standard' MC,

- multi-compartment spherical mean (MC-SM) and

- multi-compartment constrained spherical deconvolution (MC-CSD).

The general formulations of the MC and MC-SM models are as follows:

$$
\mathrm{MC}: E(\tilde{g})=\sum_{i=1}^{N} C_{i}(\tilde{g} ; \mathbf{x}), \quad \mathrm{MC}-\mathrm{SM}: \int_{\mathbb{S}^{2}} E(\tilde{g}) d \mathbf{n}=\sum_{i=1}^{N} \int_{\mathbb{S}^{2}} C_{i}(\tilde{g} ; \mathbf{x}) d \mathbf{n}
$$

where $C(\tilde{g} ; \mathbf{x})$ represents any (dispersed and/or distributed) compartment model shown in Fig. 2. ( $(\tilde{g})$ is shorthand for all PGSE acquisition parameters and $\mathbf{x}$ for all biophysical model parameters. In MC-CSD, the biophysical models are not directly summed to approximate the signal, but constitute the convolution kernel that convolved with a (model-wise) Fiber Orientation Distribution (FOD):

$$
\operatorname{MC-CSD~:~} E(\tilde{g})=\sum_{i=1}^{N} C_{i}(\tilde{g} ; \mathbf{x}) *_{\mathbb{S}^{2}} F O D_{i}(\mathbf{n}) .
$$

More details about the MC, MC-SM and MC-CSD models are given in section3. The associated generated models are then fit to data using different optimizers. 
Alimi et al.

\subsection{Modular Optimization algorithms}

Based on Dmipy's modular philosophy, each modeling framework also has a modular selection of optimization algorithms. Because of their structural similarity, MC and MC-SM models share the same optimizer choices (brute2fine and mix), while MC-CSD has algorithms specialized for estimating FODs.

brute2fine The "brute2fine" optimizer uses of scipy's brute force and L-BFGS$\mathrm{B}$ algorithms for constrained optimization of the MC parameters without needing an initial guess.

mix Dmipy also includes a generalized implementation of the recent Microstructure Imaging in Crossing (MIX) optimizer [35, which is useful when fitting highly complex models.

csd For MC-CSD models, Dmipy provides a completely generalized multi-shell constrained spherical deconvolution approach similar to Jeurissen et al. (2014) [34. For any kernel composition, it allows to enforce unity and positivity constraints on the volume fractions and FOD respectively, as well as smoothness.

\section{Reproducible Research with Dmipy}

The main purpose of Dmipy is to help increase research reproducibility in diffusion Microstructure Imaging. Dmipy is agnostic about model design, giving the user the freedom to easily create any model which is applicable to their research question, and fit it to the data PGSE data they have with the optimizer they want. Here is presented a basic overview of how to design microstructure models with the combination of biophysical models presented in the previous section.

To set up a Microstructure model in Dmipy, a 'MultiCompartmentModel' object is created by combining one or more biophysical models to represent tissue features such as water diffusivity, axon diameter distributions, axon dispersion, axon bundle crossings and extra-cellular diffusion. A constructed model object can also include custom or known parameter constraints such as axon tortuosity [26]. The following code snippet illustrates the generation of Ball and Stick [15] model in all three estimation frameworks presented in Section 2.3 .

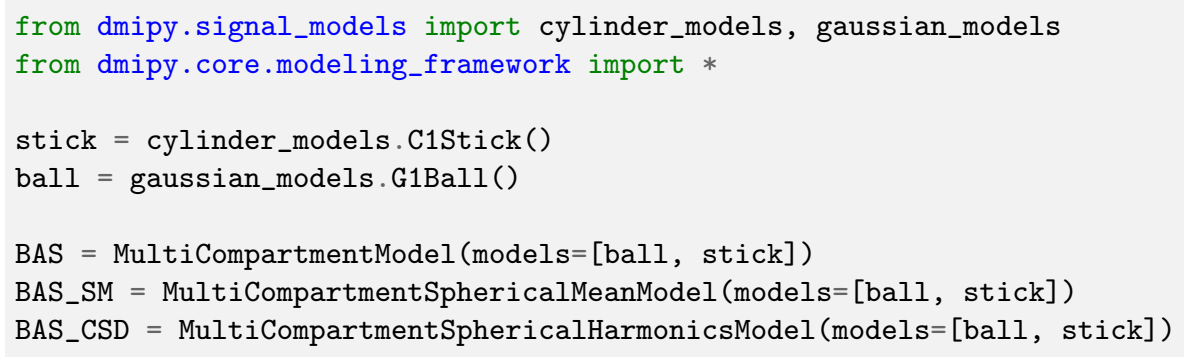

The three different MC-models generated above each use different representations of biophysical models: 'standard' for MC, 'spherical mean' for MC-SM 
and 'rotational harmonics' for MC-CSD. Every model has these representations, meaning nearly any combination of cylinders, spheres, Gaussians and distributed models (see Section 4.2) can be combined in any MC representation. Note that the MC representation is independent of any data which is to be fitted.

Fitting a Dmipy model requires only a description of the acquisition parameters (gradient strength, directions, pulse length/duration, TE) and an ND-array of dMRI data. In this way, Dmipy's fitting interface is designed very similar to dipy [33] and other successful packages. After fitting any fitted parameters, fitting errors, FODs and others can be easily recovered from the fitted model object:

BAS_fit = BAS.fit (scheme, data, solver='brute2fine')

BAS_fit.fitted_parameters \# also mse, fod, R2, etc.

The modular design of Dmipy provides the users with the means to explore their diffusion data using any model and global optimization approaches. This modularity also enables them to easily adapt/extend and also share their model implementations in their work, allowing for the addition of new models or optimizers, thus improving the reproducibility of Microstructure Imaging research.

\section{Dmipy Advanced Examples}

Dmipy can be used for a large range of applications, from modular design of multi-compartment models to signal simulation and tissue feature estimation, using usually less than 10 lines of code. In this section, we show three advanced examples of highly complex modeling approaches which are made easy using Dmipy.

\subsection{MC-SM to MC-CSD with voxel-varying kernel}

During the 2017 ISMRM: TraCED robust tractography challeng $\notin^{4}$ the winning results were obtained using CSD with a voxel-varying convolution kernel, estimated using MC-MDI[10] (an MC-SM technique). Such a method using a chain of multiple estimation frameworks is tricky to implement, yet using Dmipy it can be done with little effort, see Fig. 3

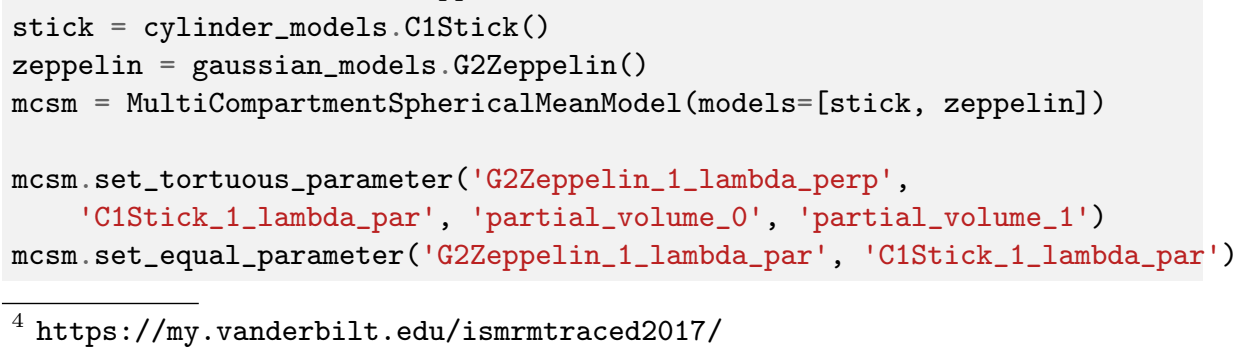



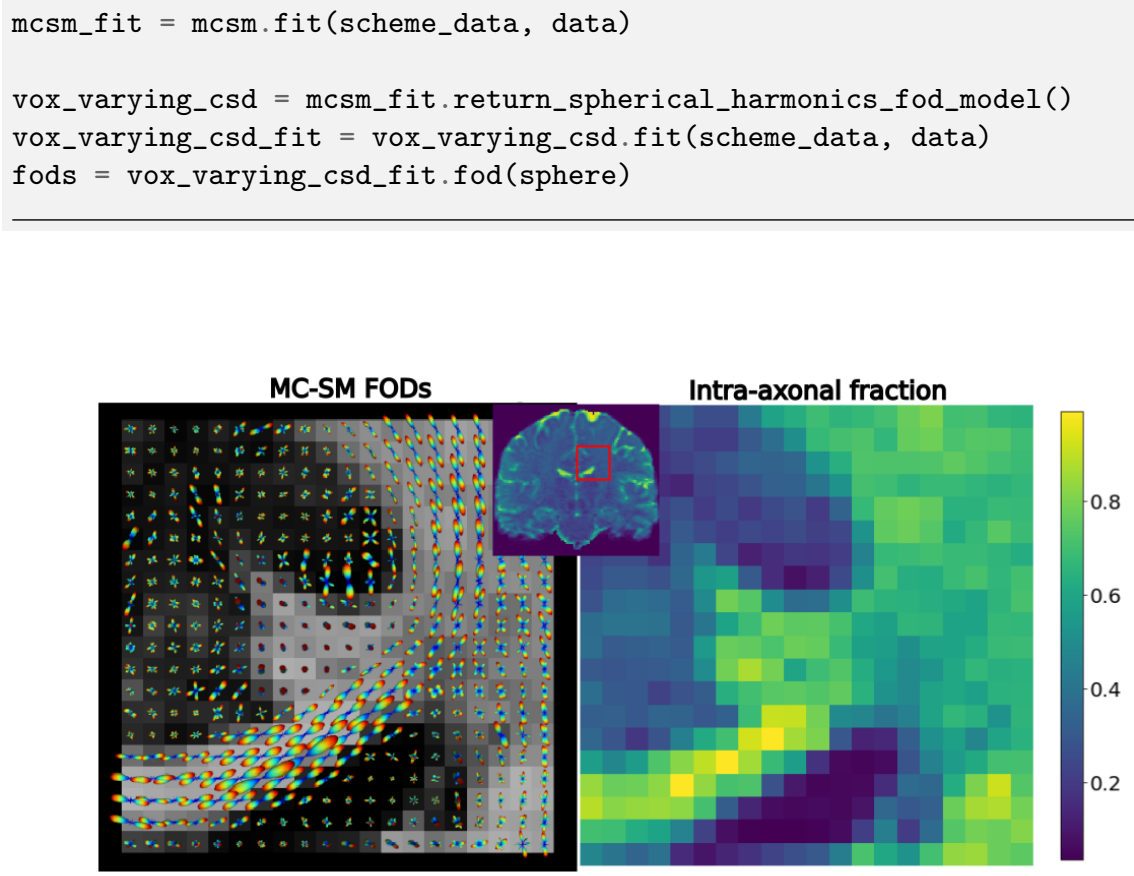

Fig. 3: Estimated FODs and intra-axonal water fraction through MultiCompartmentSphericalMeanModel.

\subsection{A "Complete" White Matter Representation}

Recent works consider axon-dispersion and the axon-diameter-distribution to be relevant phenomena for characterizing the white matter [27 21 30, as well as the temporal dependence of the extra-axonal space [24. Modeling all these effects together in one representation, with all its parameters, is quite complicated. Using Dmipy, even creating such a complicated model is very intuitive by just applying distribution effects one after another:

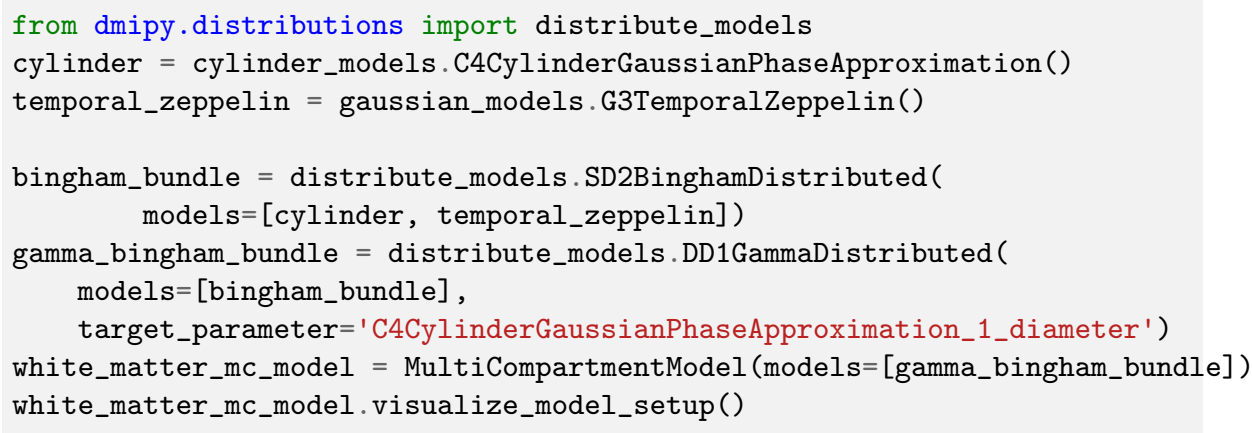


Note that despite its complexity, this model can be used to fit and simulate data like any other 5 and can even be used as a CSD convolution kernel. Line 11 can be used to visualize any model as a graph such as Fig. 4

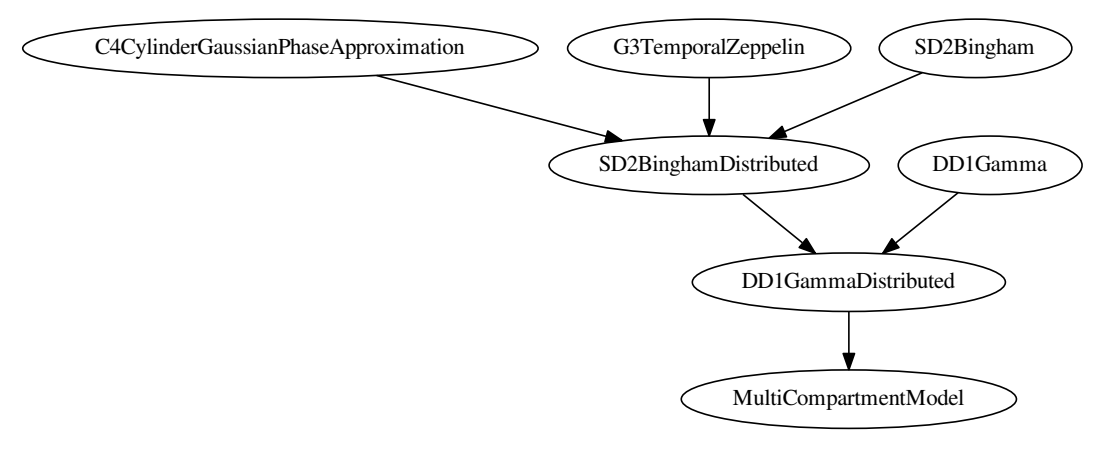

Fig. 4: Graph representation of the constructed complete white matter model.

\subsection{Multi-Tissue CSD and Multi-Tissue SMT}

Recent FOD estimation methods such as Multi-Tissue CSD (MT-CSD) obtain more robust tractograms using use non-parameters tissue response kernels data-estimated kernels representing white/grey matter and CSF 34. Dmipy can estimate these kernels directly from the dMRI dataset using the method of [16] and represents them as standard biophysical models, meaning they can be used together with parametric models by all three estimation frameworks. In the following code snippet, we show the Dmipy code to estimate tissue response models and construct both MT-CSD and multi-tissue spherical mean representation. We show the tissue volume fractions of both in Fig. 5 .

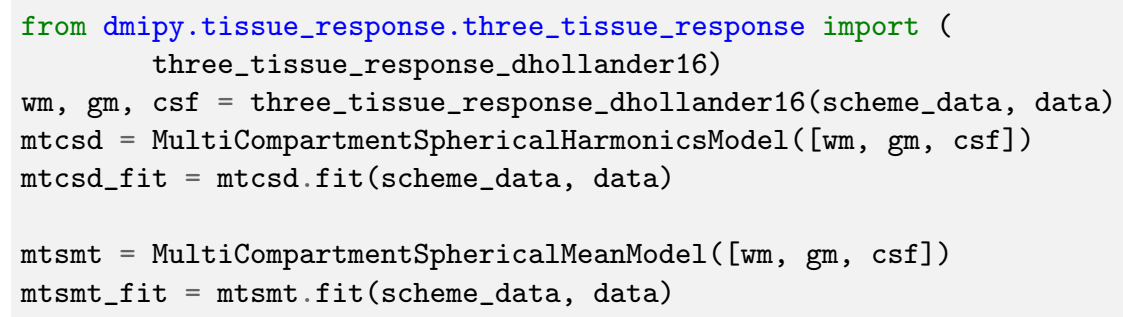

$\overline{5}$ although finding a unique minimum will be difficult given its many parameters. 


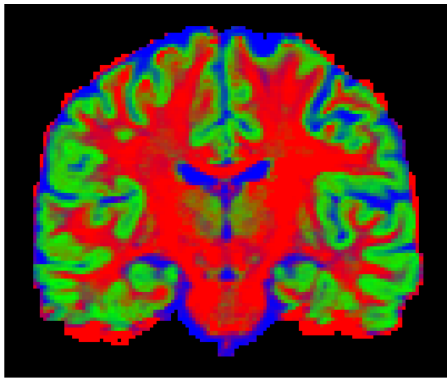

(a) Multi-Tissue CSD

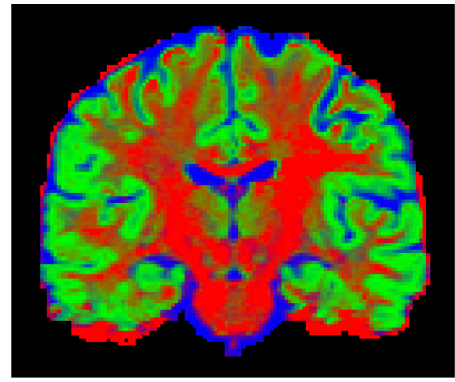

(b) Multi-Tissue SMT

Fig. 5: White Matter, Grey Matter and CSF volume fractions, estimated using (a) MT-CSD and (b) MT-SMT.

\section{Discussion}

This work introduces Dmipy, a software toolbox that allows to design multi-compartment diffusion models and to estimate their parameters. It is an open source project already available online. Therefore, users can easily tailor the software to investigate their own research and also to reproduce the models presented in the Microstructure Imaging literature.

Future work will target the implementation of a generalized gradient waveform like in MISST 3|2 to account for sequences such as STEAM or OGSE best suited for achieving, for instance, long and short diffusion times [23|24 36]. We will also compare Dmipy's performance in terms of computation time and fitting-quality with cuDIMOT [4] and MDT [5] diffusion toolboxes.

Dmipy provides many advantages. Indeed, it is an easy way to set up microstructure models (few lines of codes) with a high-level interaction. It also proposes different global optimizers including MIX 35. Dmipy is, to our knowledge, the only software solution that allows different and complementary microstructure estimation strategies (Spherical Mean, and generalized CSD, etc) for all tissue models while taking into account or not their dispersion and/or distributions. It is different from dipy 33 because Dmipy is a "model generator". That is, it does not hard-code various models. However, one can generate all existing models on the fly. Furthermore, thanks to its modular design, it is very easy to add new optimization algorithms or tissue models to be used in the framework, thus helps improve reproducibility in Microstructure Imaging research.

\section{Acknowledgments}

This work was partly supported by ANR "MOSIFAH" under ANR-13-MONU-0009-01, the ERC under the European Union's Horizon 2020 research and innovation program (ERC Advanced Grant agreement No 694665:CoBCoM). 


\section{References}

1. Panagiotaki, E., et al.: Compartment models of the diffusion MR signal in brain white matter: a taxonomy and comparison. NeuroImage 59.3: 2241-2254 (2012)

2. Ianus, A., Alexander, D.C. and Drobnjak, I.: Microstructure imaging sequence simulation toolbox. In International Workshop on Simulation and Synthesis in Medical Imaging (pp. 34-44). Springer, Cham. (2016)

3. Drobnjak, I., et al.: The matrix formalism for generalised gradients with timevarying orientation in diffusion NMR. Journal of Magnetic Resonance 210.1: 151-157 (2011)

4. Hernandez-Fernandez, M., Reguly, I., Jbabdi, S., Giles, M., Smith, S. and Sotiropoulos, S.N.: Using GPUs to accelerate computational diffusion MRI: From microstructure estimation to tractography and connectomes (2018)

5. Harms, R.L., et al.: Robust and fast nonlinear optimization of diffusion MRI microstructure models. Neuroimage, 155, pp.82-96 (2017)

6. Tournier, J.D., Calamante, F., Connelly, A.: Robust determination of the fibre orientation distribution in diusion mri: non-negativity constrained super-resolved spherical deconvolution. NeuroImage 35, 14591472 (2007)

7. Fick, R., Wassermann, D., Deriche, R.: Mipy: An Open-Source Framework to improve reproducibility in Brain Microstructure Imaging. OHBM 2018 - Human Brain Mapping, Jun 2018, Singapore, Singapore. pp.1-4. <hal-01722146>(2018)

8. Fick, R., Deriche, R., Wassermann, D.: Dmipy: An Open-source Framework for Reproducible dMRI-Based Microstructure Research (Version 0.1). Zenodo. (2018) http://doi.org/10.5281/zenodo.1188268

9. Stejskal, E., Tanner, J.: Spin diffusion measurements: Spin echoes in the presence of a time-dependent field gradient. Journal of Chemical Physics 42, 288292 (1965)

10. Kaden, E., Kelm, N.D., Carson, R.P., Does, M.D., Alexander, D.C.: Multicompartment microscopic diffusion imaging. NeuroImage (2016)

11. Wedeen, V.J., Hagmann, P., Tseng, W.Y.I., Reese, T.G., Weisskoff, R.M.: Mapping complex tissue architecture with diffusion spectrum magnetic resonance imaging. Magnetic Resonance in Medicine 54, 13771386 (2005)

12. Callaghan, P. T.: Pulsed-gradient spin-echo NMR for planar, cylindrical, and spherical pores under conditions of wall relaxation. Journal of magnetic resonance, Series A, 113(1), 53-59 (1995)

13. Assaf, Y., Freidlin, R.Z., Rohde, G.K., Basser, P.J.: New modeling and experimental framework to characterize hindered and restricted water diffusion in brain white matter. Magnetic Resonance in Medicine 52, 965978 (2004)

14. Kroenke, C. D., Ackerman, J. J.H., Yablonskiy, D.A.: On the nature of the NAA diffusion attenuated MR signal in the central nervous system. Magnetic Resonance in Medicine, 52: 1052-1059 (2004)

15. Behrens, T., Woolrich, M., Jenkinson, M., Johansen-Berg, H., Nunes, R., Clare, S., Matthews, P., Brady, J., Smith, S.: Characterization and propagation of uncertainty in diffusion-weighted mr imaging. MRM 50, 10771088 (2003)

16. Dhollander, T., et al.: Unsupervised 3-tissue response function estimation from single-shell or multi-shell diffusion MR data without a co-registered T1 image. In ISMRM Workshop on Breaking the Barriers of Diffusion MRI (Vol. 5). (2016)

17. Söderman, O., Jönsson, B.: Restricted diffusion in cylindrical geometry. Journal of Magnetic Resonance, Series A 117, 9497 (1995)

18. Vangelderen, P., DesPres, D., Vanzijl, P., Moonen, C.: Evaluation of restricted diffusion in cylinders. phosphocreatine in rabbit leg muscle. Journal of Magnetic Resonance, Series B 103, 255260 (1994) 
19. Neuman, C.: Spin echo of spins diffusing in a bounded medium. The Journal of Chemical Physics 60, 45084511 (1974)

20. Aboitiz, F., Scheibel, A.B., Fisher, R.S., Zaidel, E.: Fiber composition of the human corpus callosum. Brain research 598, 143153 (1992)

21. Assaf, Y., Blumenfeld-Katzir, T., Yovel, Y., Basser, P.J.: Axcaliber: a method for measuring axon diameter distribution from diffusion mri. MRM 59, 13471354 (2008)

22. Tariq, M., Schneider, T., Alexander, D.C., Wheeler-Kingshott, C.A.G., Zhang, H.: Binghamnoddi: Mapping anisotropic orientation dispersion of neurites using diffusion mri. NeuroImage 133, 207223 (2016)

23. Novikov, D.S., Jensen, J.H., Helpern, J.A., Fieremans, E.: Revealing mesoscopic structural universality with diffusion. Proceedings of the National Academy of Sciences, 201316944 (2014)

24. Burcaw, L.M., Fieremans, E., Novikov, D.S.: Mesoscopic structure of neuronal tracts from time-dependent diffusion. NeuroImage 114, 1837 (2015)

25. Leergaard, T.B., et al.: Quantitative histological validation of diffusion MRI fiber orientation distributions in the rat brain. PloS one 5.1: e8595 (2010)

26. Szafer, A., Zhong, J., Gore, J.C.: Theoretical model for water diffusion in tissues. Magnetic Resonance in Medicine 33, 697712 (1995)

27. Zhang, H., Schneider, T., Wheeler-Kingshott, C.A., Alexander, D.C.: Noddi: practical in vivo neurite orientation dispersion and density imaging of the human brain. NeuroImage 61, 10001016 (2012)

28. Tournier, J.D., Calamante, F., Gadian, D.G., Connelly, A.: Direct estimation of the fiber orientation density function from diffusion-weighted mri data using spherical deconvolution. NeuroImage 23, 11761185 (2004)

29. Descoteaux, M., Deriche, R., Anw, A., Bio, T., Odyssée, P.: Deterministic and probabilistic qball tractography: from diffusion to sharp fiber distributions, in: Tech. Rep. 6273, INRIA Sophia Antipolis (2007b)

30. Pizzolato, M., Wassermann, D., Deriche, R., Fick, R.: Orientation-dispersed apparent axon diameter via multi-stage spherical mean optimization. CDMRI (2018)

31. Bingham, C.: An antipodally symmetric distribution on the sphere. The Annals of Statistics , 12011225 (1974)

32. Kaden, E., Knsche, T. R., Anwander, A.: Parametric spherical deconvolution: inferring anatomical connectivity using diffusion MR imaging. NeuroImage, 37(2), 474-488 (2007)

33. Garyfallidis, E., Brett, M., Amirbekian, B., Rokem, A., Van Der Walt, S., Descoteaux, M., Nimmo-Smith, I., Contributors, D.: Dipy, a library for the analysis of diffusion mri data. Frontiers in neuroinformatics 8 (2014)

34. Jeurissen, B., Tournier, J.D., Dhollander, T., Connelly, A. and Sijbers, J.: Multitissue constrained spherical deconvolution for improved analysis of multi-shell diffusion MRI data. NeuroImage, 103, pp.411-426 (2014)

35. Farooq, H., Xu, J., Nam, J.W., Keefe, D.F., Yacoub, E., Georgiou, T., Lenglet, C.: Microstructure imaging of crossing (MIX) white matter fibers from diffusion MRI. Scientific reports. 2016 Dec 16;6:38927 (2016)

36. De Santis, S., Jones, D.K., Roebroeck, A.: Including diffusion time dependence in the extra-axonal space improves in vivo estimates of axonal diameter and density in human white matter. NeuroImage 130, 91103 (2016) 